\title{
Crítica y redención. El encuentro de las Ciencias Cognitivas con Fenomenología de la Percepción ${ }^{1}$
}

\author{
\$ Ariela Battán Horenstein \\ Instituto de Humanidades-Consejo Nacional de Investigaciones Científicas y Técnicas, \\ Universidad Nacional de Córdoba, Argentina
}

Recibido el 17/og/2020. Aceptado el 26/11/2020.

\begin{abstract}
Resumen
En este trabajo consideraré la recepción de Phénoménologie de la Perception en las Ciencias Cognitivas contemporáneas, con el objetivo de subrayar la original comprensión de la relación entre ciencia y filosofía propuesta por Merleau-Ponty en su obra. Merleau-Ponty, guiado por el lema husserliano de "volver a las cosas mismas", critica a la ciencia concebida como resultado del pensamiento objetivo. En su opinión, la ciencia privilegia la explicación sobre la descripción y oculta así, debajo de sus constructos teóricos, la validez originaria de la experiencia vivida. Sin embargo, en la comprensión merleau-pontyana de la percepción está también implicada la ciencia como fuente de material para la investigación fenomenológica. A la luz de estas consideraciones sobre el papel desempeñado por la ciencia en la comprensión fenomenológica de la experiencia, me propongo reconsiderar dos lecturas críticas dirigidas contra la comprensión del legado merleau-pontyano en el campo de investigación de las Ciencias Cognitivas. La primera de ellas es desarrollada por G. B. Madison y sus críticas apuntan al logocentrismo implicado en las Ciencias Cognitivas. La segunda es sostenida por C. Pollard para quien las Ciencias Cognitivas malinterpretan el proyecto merleau-pontyano al ignorar el objetivo trascendental que la motiva.
\end{abstract}

Palabras clave: Merleau-Ponty, fenomenología, ciencia, trascendental, logocentrismo.

Criticism and redemption, Cognitive Sciences meet Phenomenology of Perception

\footnotetext{
Abstract

In this paper I will consider the reception of Phenomenology of Perception in contemporary Cognitive Sciences. My aim here consists in emphasizing the original

1 Este artículo se enmarca dentro del Proyecto "Experiencia y Lenguaje, SECYT, Universidad Nacional de Córdoba y del Proyecto "Fenomenología del cuerpo y análisis del dolor II" (FFI2017-82272-P) del Instituto de Filosofía, CSIC, financiado por el Ministerio de Industria, Economía y Competitividad, España. Agradezco a las/los evaluadores anónimos por sus interesantes sugerencias y comentarios. He intentado integrarlos en esta versión final del trabajo.
} 
understanding of the relationship between science and philosophy offered by Merleau-Ponty in his work. Merleau-Ponty, guided by the Husserlian call "back to the things themselves", criticizes science conceived as a result of objective thought. In his opinion, science privileges explanation over description and hides under its theoretical constructions the primary validity of lived experience. However, in the merleaupontyan comprehension of perception science is also involved as a source of material for phenomenological research. Under the light of this presupposition about the role played by science in phenomenological account of experience, my paper re-evaluate two critical lectures addressed against the comprehension of the merleau-pontyan legacy in the Cognitive Sciences research field. The first one is developed by G. B. Madison and his critiques aim at the Logocentrism implied in the Cognitive Sciences understanding of human cognition. The second is sustained by C. Pollard for whom Cognitive Sciences misread the project of Merleau-Ponty ignoring its transcendental goal.

Keywords: Merleau-Ponty, Phenomenology, Science, Trascendental, Logocentrism.

El presente trabajo tiene como motivación celebrar la vitalidad de un pensador y sus ideas expuestas y desarrolladas en una obra setenta y cinco años atrás. Phénoménologie de la Perception es la obra y M. Merleau-Ponty su autor. Contra la perspectiva del museo que busca en el pasado la obra acabada y la palabra dicha para siempre, esta mirada retrospectiva estará guiada por un tema que resulta incluso hoy controversial, en torno al cual se debaten no solo fidelidades y traiciones, sino también la actualidad o la eternidad del filósofo y la obra. Me refiero concretamente al interés suscitado por Phénoménologie de la Perception (1945) en el campo disciplinar de las Ciencias Cognitivas contemporáneas y la apropiación desde allí de ciertas ideas centrales de la fenomenología de Merleau-Ponty para integrarlas en explicaciones naturalizadas de la experiencia y la conciencia (H. Dreyfus, F. Varela, S. Gallagher).

Me interesa considerar aquí dos voces críticas de alerta sobre las lecturas provenientes de las Ciencias Cognitivas, con el objetivo de esclarecer su alcance y matizar la condena a la que arriban en sus conclusiones. Estas críticas se encuentran expuestas en el artículo de G. B. Madison "Merleau-Ponty et la déconstruction du logocentrisme" (1990) y de C. Pollard "Merleau-Ponty and the Embodied Cognitive Science" (2014). Por su diferencia temporal resulta interesante comparar las críticas y verificar cómo ha cambiado, hacia el interior, la disciplina conocida como Ciencias Cognitivas y, junto con ella, el interés por la filosofía merleau-pontyana. Madison, influenciado por el optimista desarrollo de la Inteligencia Artificial de los tempranos años ‘ 90 , contrapone la comprensión merleau-pontyana de la ambigüedad de la experiencia a los intentos reductivos de las Ciencias Cognitivas. Pollard, por su parte, critica los presupuestos objetivistas que inspiran la lectura de Fenomenología de la Percepción y traicionan el proyecto trascendental de Merleau-Ponty.

El tratamiento de esa relación tendrá por objetivo algo que el propio Merleau-Ponty reclama como recaudo metodológico cuando se consideran la obra y el pensamiento de filósofas o filósofos, como él mismo, cuya voz se alza en el pasado pero todavía resuena con nitidez en el presente.

Entre una historia de la filosofía 'objetiva' que mutilara a los grandes filósofos de lo que han dado a pensar a los demás, y una meditación disfrazada de diálogo, en la que nosotros mismos haríamos las preguntas y las respuestas" resulta imperioso garantizar "... un término medio, en el que el filósofo del que se habla y el que habla estén presentes, juntos [...] (Merleau-Ponty, 1964: 195). 
Consideraré, en primer lugar, la relación del proyecto fenomenológico de MerleauPonty con el "pensamiento objetivo", luego revisaré el alcance de la crítica de Madison al modelo logocéntrico imperante en el modelo científico imperante, y los presupuestos de la crítica de Pollard en la medida en que se encuentra implicada allí una noción insuficientemente esclarecida de "trascendental". Por último, esbozaré el cambio del paradigma de las Ciencias Cognitivas que se observa entre el trabajo de Madison y el de Pollard y cuál es el interés que anima la lectura de Fenomenología de la Percepción en un contexto y otro. Concluiré este trabajo señalando algunos aspectos de lo que entiendo puede ser evaluado como ganancia teórica, también para la comprensión de la obra del filósofo, en la relación entre Merleau-Ponty y las Ciencias Cognitivas.

En 1951 Merleau-Ponty escribe "Titres et Travaux. Projet d' Enseignement", un documento destinado a dar cuenta de su labor filosófica para aspirar a una candidatura en el Collège de France. Ese texto, escrito seis años después de publicada Phénoménologie de la Perception, resulta fundamental para ponderar la motivación del filósofo una vez concluido el esfuerzo intelectual que tuvo por resultado esa obra. Merleau-Ponty manifiesta en "Titres et Travaux" su interés por hallar una tercera dimensión en la cual la reflexión que aspira a comprender al hombre pueda superar las alternativas del sujeto y el objeto y de la primera y la tercera persona que caracterizan la disputa por el sentido (o más bien por la determinación de éste) entre la filosofía y el saber positivo, es decir, la ciencia. Lejos de pretender una resolución reduccionista al conflicto, Merleau-Ponty reclama un medio común para la filosofía y la ciencia, superador tanto de una ciencia empírica ciega, como, también, de una "conciencia filosófica que desconoce su nacimiento" (Merleau-Ponty, 2000: 12). Así, lejos de renunciar a la aspiración científica para su propuesta, denuncia los devastadores efectos de toda forma de reduccionismo en la comprensión de la experiencia. Esto sirve de fundamento, además, de esa búsqueda de una tercera dimensión "donde nuestra actividad y nuestra pasividad, nuestra autonomía y nuestra dependencia, dejarían de ser contradictorias" (Merleau-Ponty, 2000: 13). El reclamo por un medio común para la filosofía y la ciencia como proyecto epistemológico que recorre Fenomenología de la Percepción y articula el tratamiento de cada una de las temáticas (fisiología, psicología, lingüística, historia) requiere de una equilibrada crítica a las pretensiones del pensamiento objetivo, pero sin olvidar o pasar por alto los requisitos de rigor científico y pertinencia de la investigación fenomenológica. "Se trata de describir, no de explicar ni analizar", sentencia Merleau-Ponty en el Prólogo a Fenomenología de la Percepción (1985), donde también reivindica el llamado de Husserl a "volver a las cosas mismas" en cuanto recusación de la ciencia, entendida esta como un intento de sustitución o suplantación de la experiencia por una versión "explicada" y, en ese sentido, empobrecida de la misma. "Volver a las cosas mismas es volver a este mundo antes del conocimiento del que el conocimiento habla siempre, y respecto del cual toda determinación científica es abstracta, signitiva y dependiente..." (MerleauPonty, 1985: 9). La primacía de la experiencia es propuesta, en consecuencia, no como alternativa escéptica a la validez de la ciencia, sino más bien, como su fundamento, en la medida que le proporciona el objeto sobre el cual aquella versa, pero también ofrece las indicaciones metodológicas para su proceder. En ese sentido, es posible afirmar que Merleau-Ponty inaugura en Fenomenología de la Percepción una tercera

2 Resuena en esas reflexiones el eco de la lectura que Merleau-Ponty hiciera de los manuscritos de La Crisis de las Ciencias Europeas y la Fenomenología Transcendental, a los que tuvo acceso apenas unos meses después de que Van Breda pusiera a salvo el legado husserliano en Lovaina. 
vía epistemológica que funda en la experiencia de los fenómenos, en el estrato precientífico, la validez de las operaciones de la ciencia. Afirma,

Todo el universo de la ciencia está construido sobre el mundo vivido y, si queremos pensar rigurosamente la ciencia, apreciar exactamente su sentido y alcance, tendremos, primero, que despertar esta experiencia del mundo del que ésta es expresión segunda (Merleau-Ponty, 1985: 8).

Esto coloca a la fenomenología bajo el linaje de las grandes teorías del conocimiento de la modernidad, tras los pasos de Descartes y Kant, pero también en una línea hereditaria directa con la obra literaria y artística de Balzac, Proust, Valery y Cézanne. Para Merleau-Ponty el destino de la labor filosófica sintetiza así un doble mandato, por un lado, el de los grandes sistemas filosóficos modernos que proponen "aprender de nuevo a ver el mundo" $y$, por otro lado, el del arte en su tarea de "realizar una ver$\mathrm{dad}$ " antes bien que limitarse a reflejarla. Como señala R. Walton, hay una estrecha relación entre esta idea de la filosofía como realización de una verdad y la asunción de la contingencia ontológica del mundo. "Por eso el mundo no nos impone un espectáculo sino que exhibe un polimorfismo" (Walton, 2012:121) que descentra al sujeto y solo lo admite como campo o polo de irradiación, ek-stasis. Afirma Merleau-Ponty,

El mundo, cual hemos intentado mostrarlo, como unidad primordial de todas nuestras experiencias en el horizonte de nuestra vida y término único de todos nuestros proyectos no es ya el despliegue visible de un Pensamiento constituyente [...], sino la patria de toda racionalidad (Merleau-Ponty, 1985: 438).

Esto permite afirmar que, junto con su idea de la filosofía, Merleau-Ponty esboza un modelo de racionalidad que disputa el dominio de lo que G. B. Madison llama el Logocentrismo. En ese modelo de racionalidad, la tercera vía pretendida como forma de superación tanto de los reduccionismos como de los dualismos también desempeña un papel central, pues, como señala en el apartado final del Avant propos, la medida de la racionalidad es la experiencia. La noción merleau-pontyana de experiencia y sus atributos son el leitmotiv que toma Madison para emprender la crítica al Logocentrismo, es decir, a la "univocidad luminosa del logos" que se erige sobre la idea de que la "realidad es una cosa enteramente determinada en sí, completa y autosuficiente" (Madison, 1990: 66). Bajo esa denominación, el estudioso de la obra de MerleauPonty, reúne variantes del pensamiento objetivo en su versión contemporánea como, por ejemplo, las Ciencias Cognitivas y la Inteligencia Artificial. El juicio de Madison sobre estas disciplinas es lapidario, pues entiende que "sería difícil encontrar dos incomprensiones más completas de la comprensión humana. En su búsqueda del algoritmo universal, - agrega - ellas representan una suerte de enfermedad genética programada en el espíritu humano" (Madison, 1990: 74).

Si bien luego me ocuparé de señalar los cambios en el seno de las Ciencias Cognitivas que permitirán matizar esta aseveración, lo que interesa aquí considerar son las razones de las críticas de Madison. Estas están inspiradas, precisamente, en la concepción de la experiencia que Merleau-Ponty presenta en Fenomenología de la Percepcióny su recusación de la inversión filosófica de la lógica vivida que da origen y prestigio al pensamiento objetivo. Según Madison, el programa de investigación que anima el desarrollo de las Ciencias Cognitivas y la Inteligencia Artificial es una actualización del "modo naturalista (partes extra partes) de explicación que Merleau-Ponty ataca en

3 Pues como dice casi al final del Avant-Propos, "La verdadera filosofía consiste en aprender de nuevo a ver el mundo, y en este sentido una historia relatada puede significar el mundo con tanta «profundidad» como un tratado de filosofía." (Merleau-Ponty, 1985: 20). 
Estructura del Comportamiento y en la Fenomenología de la Percepción" y "está más vivo y extendido que nunca" (Madison, 1990: 75). Una referencia teórica a la que alude Madison en su trabajo es la temprana obra de H. Dreyfus, What computers Can't Do.A Critique of Artificial Reason de 1972. En esa obra Dreyfus incluye a Merleau-Ponty entre los filósofos que ofrecen perspectivas "alternativas" a la comprensión de la cognición en términos computacionales. Con el paso del tiempo, y el desarrollo de las Ciencias Cognitivas, la aparente sintonía crítica entre Madison y Dreyfus se desvanecerá. Para Dreyfus, el pensamiento merleau-pontyano resultará un eficaz complemento en la formulación de modelos explicativos de la cognición que logren superar los obstáculos del cognitivismo (reduccionismo de la conciencia al modelo de la computadora o al cerebro, la tesis de un sujeto cognitivo desencarnado y descontextualizado y la comprensión de la cognición como manipulación lógica de símbolos, aplicación de reglas y elaboración de representaciones discretas del mundo externo). En esa misma dirección también, Dreyfus avanzará en sus trabajos posteriores para poner en continuidad, desde una perspectiva naturalizada, la intencionalidad corporal merleau-pontyana (sin conceptos ni representaciones) y la comprensión de la dinámica cerebral en el contexto de redes neuronales simuladas. ${ }^{4}$ Sin embargo, Madison bajo una motivación semejante, esta es, el reconocimiento del origen corporal y perceptivo de las "formaciones superiores de la conciencia" (Madison, 1990: 67) en el pensamiento merleau-pontyano, recusará las pretensiones de las Ciencias Cognitivas. En particular el proyecto de "traducir la experiencia humana en lenguajes formales, lógicos (los únicos que una computadora es capaz de 'comprender')", en los cuales no hay lugar "para la ambigüedad del sentido", resulta inaceptable para Madison. "La machina rationatrix que es la computadora - agrega - es una encarnación viva del principio estéril de identidad, la 'no contradicción estéril de la lógica formal', como dice Merleau-Ponty" (Madison, 1990: 75).

En su crítica, Madison no se reserva descalificaciones hacia las Ciencias Cognitivas, se refiere a ellas en términos de "incomprensión", "enfermedad genética", "empresa iniciada sobre una pista falsa", "peligrosa", insuficiente e inverosímil, que "no puede enseñarnos nada sobre las formas de comprensión típicamente humana" (Madison, 1990: 75). Las razones que sustentan su posición son, en concreto, la convicción de que la comprensión humana se resiste a todo intento de formalización, y la consideración del carácter fundamentalmente ambiguo e indeterminado de la experiencia. Para Madison, siguiendo en ello a Merleau-Ponty, hay un fondo tácito, incomprensible en términos formales, que caracteriza a la cognición humana y al modo en que comprendemos el "mundo". Este mundo, el de la experiencia vivida, se diferencia del "universo" entendido como concepto de la lógica, es decir, como dominio o conjunto de cosas, precisamente porque siempre guarda un fondo no tematizable. Según Madison, tanto los proyectos de formalización como también la búsqueda de un modelo cibernético para la mente o la aspiración a un lenguaje unívoco universal están mal orientados, pues se dirigen hacia una quimera metafísica. Madison recupera así el, tantas veces revisitado, epíteto que caracteriza la intención filosófica que anima Fenomenología de la Percepción. Para Merleau-Ponty, afirma Madison,

[...] la noción de 'ambigüedad' o de 'indeterminado' es esencial para la comprensión de la manera en la cual creamos el sentido en nuestras vidas, para la comprensión, en consecuencia, de la 'trascendencia humana', uno de los temas más importantes de la Fenomenología (1990: 78) 
La trascendencia humana no es más que otro nombre para la noción de existencia, de la experiencia vivida, la cual, como subraya Madison, es irreductible a cualquier forma de naturalismo.

Ahora bien, ¿resulta fértil en términos teóricos la posición de Madison?, ¿constituye su perspectiva un aporte a la comprensión del pensamiento de Merleau-Ponty o del alcance y pretensiones de las Ciencias Cognitivas? En mi opinión, la crítica de Madison se asemeja más a una queja impotente que se invalida a sí misma, antes bien que a una crítica productiva en sentido filosófico. Bajo una caracterización radical de la irreductibilidad de la comprensión humana, Madison rechaza, como vimos, todo intento de reconstrucción de la experiencia que pueda provenir de lo que él denomina el logocentrismo. En ese sentido, lo estéril de su crítica residiría en la alternativa maniquea sobre la cual se funda. Madison contrapone, sin matices, trascendencia y existencia a logocentrismo y formalización, sin tener en cuenta, en consecuencia, la complejidad de la propuesta merleau-pontyana acerca de la relación entre lo vivido (experiencia) y lo conocido (pensamiento objetivo). Por otro lado y sin pretender con ello incurrir en anacronismos indeseados, es posible señalar como dato contextual, que el propio desarrollo disciplinar de las Ciencias Cognitivas (sobre el cual me detendré más adelante) contribuye de manera efectiva en la relativización de los aspectos más criticados por Madison de ese acercamiento disciplinar a Merleau-Ponty. Resultado de una "feliz coincidencia", las propias Ciencias Cognitivas se vuelven críticas de la "quimera metafísica" (en términos de Madison) que supone pretender explicar la conciencia humana como si fuera una computadora y la experiencia como intercambio semántico de información.

Apoyado en una lectura sesgada de Fenomenología de la Percepción, Madison no logra captar y mostrar la equilibrada posición que Merleau-Ponty reclama respecto de la relación entre ciencias empíricas y descripción de la experiencia vivida, o lo que he llamado, la apuesta por una epistemología fenomenológica de la tercera vía.

\section{II}

La perspectiva epistemológica que atraviesa Fenomenología de la Percepción asume el hecho de que la "conciencia científica toma prestado a las estructuras de la experiencia vivida todos sus modelos", pero sin explicitar "los horizontes de conciencia perceptiva" (Merleau-Ponty, 1985: 79) que la rodean. Los horizontes delimitan un campo fenomenal y despejan la posibilidad de fundar esa pretensión epistemológica en "una subjetividad trascendental autónoma situada en todas partes" (Merleau-Ponty, 1985: 83). A estas conclusiones llega Merleau-Ponty de la mano de la psicología en el primer apartado de su obra, se trata de una psicología en sentido propio que apunta, a través de la revisión del significado y alcance de las nociones de sensación, atención y juicio, al núcleo central de la experiencia cognitiva y perceptiva. La alusión de Merleau-Ponty a esta disciplina no debería ser pasada por alto en este contexto, porque, más allá de las discusiones acerca de exactamente cuál sea el referente de "la psicología" a la que dirige sus críticas, resulta muy esclarecedor el modelo de investigación o ciencia empírica de la conciencia a que esa caracterización remite. La psicología ofrece para Merleau-Ponty un ejemplo de investigación motivada fenomenológicamente que avanza desde la actitud natural a la actitud trascendental. El psicólogo es presentado así como una suerte de sujeto epistemológico modelo al que hay que seguirle los pasos, pero no por el saber sobre el "hombre" o la "conciencia" que pueda ofrecer, sino, más bien, con el objetivo de recuperar la comunicación interior con el mundo que es la experiencia. Por eso, afirma que la psicología va a parar siempre al problema de la constitución del mundo. El estudio de la conciencia sobrepasa los límites de 
los postulados del sentido común y la psicología, aún desde una posición realista e ingenua, confronta al investigador con un interrogante más radical.

Si, por ejemplo, nos proponemos hacer una psicología positiva de la percepción [...] nos vemos obligados a describir el objeto y el mundo tal como aparecen a la conciencia y, por ende, a preguntarnos si este mundo inmediatamente presente, el único que conozcamos, no es así mismo el único del que quepa hablar (MerleauPonty, 1985: 80).

El interrogarse radical es, para Merleau-Ponty, la apertura del campo trascendental en el que la fenomenología se desenvuelve, pero precavida ya del error de las filosofías trascendentales clásicas que dan por hecho aquello que deberían explicitar. MerleauPonty entiende que una filosofía "se vuelve trascendental [...] no instalándose en la conciencia absoluta sin mencionar los procedimientos que a la misma conducen, sino considerándose a sí misma como problema..." (Merleau-Ponty, 1985: 84).

Estas consideraciones epistemológicas que en lo inmediato me permiten introducir una segunda crítica dirigida a ese campo disciplinar en la cual, nuevamente, se apela a Fenomenología de la Percepción, darán ocasión luego de presentar la opinión sobre la relación de las Ciencias Cognitivas y la fenomenología merleau-pontyana que anima este trabajo.

El artículo de C. Pollard, "Merleau-Ponty and Embodied Cognitive Science", como su título lo anticipa, es un intento de reconstrucción de las lecturas emblemáticas que autores, enrolados en la corriente pos-cognitivista y corporeizada, hacen de aspectos de Fenomenología de la Percepción, en la búsqueda de fundamento teórico a sus posiciones. H. Dreyfus, S. Gallagher, y los autores de De cuerpo presente. Las ciencias cognitivas y la experiencia humana, F. Varela, E. Thompson y E. Rosch, son los autores convocados por Pollard con el objetivo de confrontar sus opiniones, guiadas por intereses foráneos, con el estudio exegético de la obra. En ese sentido es posible reconocerle a Pollard su esfuerzo pues ofrece todos los argumentos que quienes estudiamos al filósofo deseamos atender. En primer lugar, destaca que el interés de estos autores por la obra de Merleau-Ponty no ha estado guiado por una preocupación exegética y, en segundo lugar, que ese interés ha estado, más bien, motivado por la aparente convergencia entre las descripciones fenomenológicas de Merleau-Ponty y los resultados de las Ciencias Cognitivas contemporáneas. A partir de esto organiza su presentación, que va a ser crítica respecto de estos intentos por hacer converger perspectivas y metodologías, en torno a un interrogante: “¿qué habría pensado el Merleau-Ponty de Fenomenología de la Percepción de este uso de su fenomenología en las Ciencias Cognitivas?" (Pollard, 2014: 69). La respuesta que plantea Pollard contempla un aspecto fundamental del pensamiento merleau-pontyano, que en este trabajo también me he ocupado de destacar, el interés por las ciencias empíricas como base a partir de la cual se origina y orienta la investigación fenomenológica. En consecuencia afirma, "hay pocas dudas de que [Merleau-Ponty] sería entusiasta respecto del proyecto de las Ciencias Cognitivas como una herramienta para profundizar nuestra comprensión de los detalles y complejidades de la conciencia" (Pollard, 2014: 69). Sin embargo, Pollard no se contenta con esto porque logra entrever que esa coincidencia en el interés científico, obstaculiza la captación "del profundo significado filosófico que él [Merleau-Ponty] buscaba expresar" (Pollard, 2014: 69). 
La posición de Pollard distingue, a los fines de presentar sus críticas a la apropiación conceptual de Dreyfus, Gallagher y Varela et al., el interés de Merleau-Ponty por la investigación empírica de las ciencias, de su significación eminentemente filosófica, o lo que aquí he denominado epistemológica, y que el autor del trabajo denomina "ontológica". La estrategia de Pollard consistirá entonces en mostrar que los intentos de conciliar la fenomenología merleau-pontyana y las Ciencias Cognitivas traicionan los propios límites propuestos por el filósofo para evitar que se inmiscuya el pensamiento objetivo en la comprensión de la experiencia. En opinión de Pollard, "estos usos de Merleau-Ponty tácitamente des-trascendentalizan su fenomenología" (Pollard, 2014: 69) con el propósito de adecuarla a los objetivos disciplinares de las Ciencias Cognitivas. La crítica de Pollard concretamente apunta al hecho de que las Ciencias Cognitivas subordinarían las motivaciones filosóficas (ontológicas) a las motivaciones empíricas. La operación de "des-trascendentalización" la reconoce Pollard en el tratamiento que los autores estudiados hacen de tres nociones centrales del pensamiento merleau-pontyano, más precisamente, de la obra del '45: "arco intencional" (Dreyfus), "esquema corporal" (Gallagher) y "cuerpo vivido" (Varela, Thompson y Rosch). El núcleo de la crítica de Pollard es, en consecuencia, que la traducción de esas nociones a un esquema naturalizado de comprensión, que está en continuidad con las ciencias empíricas, convierte a la fenomenología de Merleau-Ponty en psicología fenomenológica, es decir, una ciencia empírica comprometida ontológicamente y circunscripta al ámbito reflexivo de la actitud natural. Bajo estas condiciones se alentaría una lectura des-trascendentalizante de la empresa filosófica merleau-pontyana a la que Pollard caracteriza como fundamentalmente trascendental. Pollard entiende por trascendental una "perspectiva filosófica que se pregunta acerca de lo que hace posible el campo fenomenal, es decir, se hace la pregunta trascendental acerca de cuáles son sus condiciones de posibilidad" (Pollard, 2014: 75).

La crítica de Pollard, sin lugar a dudas, permite poner al descubierto cuestiones centrales para los intérpretes, ya sean ortodoxos o heterodoxos, de la filosofía de Merleau-Ponty, referidas al alcance y significación de la calificación de la filosofía merleau-pontyana como filosofía trascendental ${ }^{8}$ y su relación con la empresa trascendental de Husserl, la propia definición de la noción de trascendental en el contexto del pensamiento merleau-pontyano y, en términos generales, el vínculo entre naturalización de la fenomenología y las pretensiones trascendentales que la caracterizan.

\footnotetext{
6 En rigor de verdad no es el objetivo de este trabajo someter a ningún escrutinio "la posición" de Pollard (cuyo artículo ciertamente recomiendo leer con entusiasmo por su aporte a la exégesis merleau-pontyana), eso requeriría un estudio más detallado de las tesis presentadas por él. Lo que intento es una tarea más modesta, revisar críticamente sus opiniones acerca de la relación entre fenomenología merleaupontyana y Ciencias Cognitivas. Sin embargo, no puedo dejar de mencionar que el énfasis de Pollard sobre el aspecto ontológico de las conclusiones de la investigación trascendental de Merleau-Ponty es controversial. Pollard parece subordinar la motivación fenomenológica que anima el estudio de la experiencia y dirige la atención hacia las ciencias empíricas a la "intención" explícita de elaborar una ontología. Al menos en Estructura del Comportamiento y Fenomenología de la Percepción, Merleau-Ponty evita comprometerse ontológicamente con algo que vaya más allá del aparecer fenoménico, por esta razón la postulación de la noción de chair en su última filosofía es interpretada como un giro que avanzaría de la fenomenología a la ontología. Pollard sostiene, pero sin dar fundamento textual que lo avale, que "Merleau-Ponty explícitamente afirma que tiene la tarea de formular una ontología fenomenológica (una fenomenología existencial) cuyos términos técnicos claves, 'cuerpo-sujeto' y 'ser-en-el-mundo' sirven para expresar estructuras del ser —ellos son categorías fenomenológico-ontológicas no solo fenomenológicas" (Pollard, 2014: 74). Esta presunción, sin duda, guía a Pollard en su comprensión de la dimensión trascendental de la filosofía de Merleau-Ponty.

7 Cabe aquí realizar una aclaración en relación a la noción de "naturalización" y sus variantes asociadas. El término alude en sentido laxo a la aspiración explicativa que tiene por finalidad dar cuenta de lo existente como entidades espacio-temporalmente determinadas, sometidas a la causalidad, empíricamente verificables y formalizables, en los términos que las ciencias naturales lo hacen. Así, un esquema naturalizado de explicación no depende solo del concepto de naturaleza sino también de un determinado estado de desarrollo de las llamadas ciencias naturales. La concepción mecánica de la naturaleza promueve un esquema "naturalizado" de explicación que postula la existencia partes extra partes de los objetos y relaciones de causalidad por contacto (este es, por caso, el paradigma dominante en la fisiología sobre la cual Merleau-Ponty reflexiona en el apartado "El cuerpo como objeto y la fisiología mecanicista"). En un sentido más restringido y actual la noción de "naturalización" debe ser enmarcada en el contexto del conocido Proyecto de Naturalización de la Fenomenología surgido en los años ' 90 (CF. Petitot, J., Varela, F., Pachoud, B., Roy, J.-M., 1999). En ese marco, el adjetivo "naturalizado" contempla todavía la exigencia de que las entidades y propiedades aludidas en las explicaciones de la conciencia resulten concordantes con las categorías propuestas por las ciencias naturales, aunque estas ya no se encuentren dominadas por un paradigma mecanicista. En este sentido, los intentos de traducción de las nociones de arco intencional, cuerpo vivido y esquema corporal en el vocabulario de las Ciencias Cognitivas Corporeizadas, considerados por Pollard en su trabajo, son un ejemplo de naturalización de la fenomenología merleau-pontyana. 8 CF. Gardner (2015).
} 
El enfoque de Pollard reconoce y enfatiza las diferencias sustanciales que distinguen los dos modos, el husserliano y el merleau-pontyano, de comprender el compromiso trascendental de la fenomenología. En el caso de Merleau-Ponty se trata de una filosofía trascendental no apriorística o, más bien, cuyo a priori depende de la situación, la perspectiva y la encarnación del sujeto, en definitiva se trata, según Pollard, de una versión existencial de la filosofía trascendental (Cf. Pollard, 2014: 85). El embate crítico de Fenomenología de la Percepción al compromiso ontológico objetivista de las ciencias empíricas es de naturaleza trascendental. Esto es posible, según lo comprende Pollard, por la intermediación de la reducción que es la que asegura la perspectiva trascendental. Pollard parece dar a nociones como "filosófico", "fenomenológico", y, en especial, "trascendental" una suerte de valor "honorífico". Por ello la denuncia de des-trascendentalización de la fenomenología merleau-pontyana va acompañada de una advertencia acerca del riesgo de convertir a esta en psicología fenomenológica, en definitiva una ciencia empírica.

Como réplica a Pollard se podría insistir en dos cosas, por un lado, en recuperar el sentido que el propio Merleau-Ponty reserva para la fenomenología como filosofía trascendental y, en segundo lugar, recordar que para el filósofo la reducción no es nunca una reducción completa. El carácter trascendental de la perspectiva merleau-pontyana está determinado por el cumplimiento de una serie de tareas y la delimitación de una esfera de inherencia para la fenomenología (antes bien que por la determinación de las condiciones de posibilidad): poner entre paréntesis, dejar en suspenso y comprender las tesis de la actitud natural. El término trascendental alude, entonces, al cómo antes bien que al qué. Afirma Merleau-Ponty,

Esta palabra significa que la reflexión nunca tiene bajo su mirada al mundo entero y la pluralidad de las mónadas desplegadas y objetivadas, y que solo dispone de una visión parcial y de un poder limitado. Es también por eso que la fenomenología es una fenomenología, eso es, estudia la aparición del ser en la consciencia, en lugar de suponer dada de antemano su posibilidad (MerleauPonty, 1984: 82).

En ese sentido, quizás puede señalarse que Pollard confunde sus propias intenciones filosóficas con las de Merleau-Ponty cuando afirma que,

Desde la perspectiva de Merleau-Ponty, la investigación científica juega un rol crucial ayudando a sacar a la luz y enfocar nuestra atención sobre aspectos importantes del cuerpo y la percepción a través de una metodología sistemática y rigurosa que implica determinar patrones usando percepciones dentro de la actitud natural [...] (Pollard, 2014: 88).

Ahora bien, hasta allí se podría estar de acuerdo en que en ello reside el interés de Merleau-Ponty por las ciencias empíricas, pero luego agrega Pollard que "Esos patrones empíricos, sin embargo, deberían ser integrados dentro de una teoría del ser humano para que su pleno significado filosófico pueda ser comprendido" (2014: 88).

Esta última afirmación es la que no parece hacer justicia a las pretensiones epistemológicas de Merleau-Ponty, las cuales no aspiran a una ciencia unificada, tampoco

9 Más allá del hecho de que Pollard reconoce la afinidad que Merleau-Ponty habría tenido con las Ciencias Cognitivas, resulta interesante señalar la semejanza de este argumento con algunos que se esgrimen contra el Proyecto de Naturalización de la Fenomenología. La imposibilidad de reconciliar filosofía trascendental y naturalización es uno de ellos. En este sentido se puede recurrir a la opinión de N. Depraz (1999), quien neutraliza las voces críticas. Depraz es optimista respecto de la posibilidad de integrar la fenomenología y los reportes naturalizados de las Ciencias Cognitivas, en la medida en que aquella se mantenga a salvo de la "tendencia a sustancializar el objeto (sin importar qué objeto pueda ser) apuntado en la descripción" (Depraz, 1990: 466). 
apuestan a un fundacionismo fenomenológico, sino, más bien, al desenmascaramiento de las pretensiones del pensamiento objetivo, pero, precisamente, para restablecer el vínculo con la experiencia. La fenomenología se da para sí la tarea de escoltar a las ciencias empíricas y esto es lo que desde los autores provenientes de las Ciencias Cognitivas rescatan cuando van a su encuentro, pues como señala Merleau-Ponty,

[...] ahora que el campo fenomenal se ha circunscrito suficientemente, entremos en este dominio ambiguo y demos en él, con el psicólogo, nuestros primeros pasos, esperando que la autocrítica del psicólogo nos conduzca, por una reflexión de segundo grado, al fenómeno del fenómeno y convierta decididamente en campo trascendental el campo fenomenal (Merleau-Ponty, 1984: 84). ${ }^{10}$

Si sustituimos en este fragmento "psicólogo" por "científico cognitivo", podremos redimir a las Ciencias Cognitivas y encontrar en (algunas) de sus preocupaciones e investigaciones el material para nuestras descripciones fenomenológicas.

\section{III}

Una vez relevadas y evaluadas las críticas es posible avanzar en la dirección señalada como motivación principal de este trabajo. Tal como se puso de manifiesto al comienzo del mismo, no se trata de leer de manera unilateral este intento de intercambio teórico entre fenomenología merleau-pontyana y Ciencias Cognitivas, sino, más bien, de dar lugar al diálogo que pueda resultar enriquecedor para ambas perspectivas. Es un hecho fácilmente comprobable en la literatura especializada que, en las últimas décadas, junto con una revitalización del interés por la obra de Merleau-Ponty, se ha producido una reorientación temática del mismo. ${ }^{11}$ Revisiones de las reflexiones merleau-pontyanas acerca de la distinción entre imagen y esquema corporal, el condicionamiento corporal de la cognición, la intersubjetividad como intercorporeidad no parecen ser efecto exclusivo de la exégesis especializada, en muchos casos esos impulsos vienen motivados por las lecturas que H. Dreyfus, E. Thompson, F. Varela, S. Gallagher y sus adherentes y críticos han hecho de la obra de Merleau-Ponty.

En ese sentido, la noción de diálogo debe primar en la ponderación de la relación entre fenomenología merleau-pontyana y Ciencias Cognitivas con el objetivo de contribuir, también, en la comprensión de la obra del filósofo y evitar así los juicios estériles. Entre los cuales se cuentan, tanto la condena general de las pretensiones de esa relación, como una comprensión sesgada y unidireccional de lo que sean "las" Ciencias Cognitivas. Pues, por un lado, como toda denominación colectiva alude a un conjunto de teorías, presupuestos metodológicos y líneas de investigación con programas propios e incluso divergentes entre sí, ${ }^{12}$ de los cuáles no todos son en igual medida, lo que podríamos denominar, merleauponyian friendly. Y, por otro lado, es importante tener en cuenta que a pesar de su cercanía temporal y actualidad histórica, esas teorías también evidencian un

\footnotetext{
10 Se reconocen en estas palabras ecos de la reflexión husserliana sobre la compleja relación entre psicología y fenomenología de los parágrafos 58 a 71 de La Crisis de las Ciencias Europeas y la Fenomenología Trascendental, aunque con una diferencia sustantiva. Para Husserl la esfera trascendental se abre a condición de que el psicólogo no avance. Merleau-Ponty, en cambio, propone la apertura del campo trascendental como consecuencia de un avanzar conjunto. El psicólogo, como el niño, afirma Husserl, "tiene experiencia de las cosas en tanto que cosas, [...] pero todavía no tiene idea de las estructuras internas que aún faltan totalmente en sus apercepciones de las cosas" (Husserl, 1991: 259 y s.)

11 Sobre este punto me he explayado en "Usos impropios y ganancia teórica, la lectura de Fenomenología de la Percepción desde las Ciencias Cognitivas" (de próxima aparición en un volumen del Anuario Colombiano de Fenomenología).

12 En opinión de Varela, como cualquier ciencia que se observe de cerca, "las ciencias cognitivas constituyen una diversidad de visiones semicompatibles y no un campo monolítico. Más aún, como cualquier actividad social, tiene polos de dominación, de tal manera que algunas de las voces participantes adquieren más fuerza que otras en distintos períodos de tiempo" (Varela, 2000: 182).
} 
desarrollo y una evolución en las últimas décadas. Esto resulta central, por ejemplo, para distinguir la crítica a la posibilidad de relación entre la fenomenología de Merleau-Ponty y las Ciencias Cognitivas elaborada por Madison en los noventa, en un contexto teórico dominado por el optimismo generado por la Inteligencia Artificial y la crítica de Pollard dirigida a representantes del giro enactivo y corporizado (poscognitivista o poscartesiano) en Ciencias Cognitivas en las décadas siguientes. Otro aspecto a tener en cuenta es el carácter bifronte de las Ciencias Cognitivas, como Varela, Thompson y Rosch lo han hecho notar, en cuanto se define como una conjunción híbrida de ciencias naturales y ciencias humanas.

Si bien no es este el lugar para trazar una historia de las Ciencias Cognitivas, ${ }^{13}$ tarea que ante todo desbordaría mi conocimiento de la temática, si me interesa señalar brevemente la genealogía que orienta la atención de algunas de sus líneas de investigación hacia la fenomenología de Merleau-Ponty y, en particular, a la obra de 1945. Dos preocupaciones fácilmente identificables dentro del desarrollo disciplinar de las Ciencias Cognitivas son las que parecen haber motivado el giro de la atención a lo que $\mathrm{H}$. Dreyfus llamaba maneras alternativas de comprensión de la inteligencia humana (Dreyfus, 1972). Por un lado, (i) las limitaciones del paradigma computacional para la comprensión de la cognición humana y, por otro lado, (ii) el peligro de "restringir la experiencia vivida a las neuronas" (Depraz, 1999: 474), es decir, el riesgo del reduccionismo materialista.

Dreyfus considera que dentro de esas maneras alternativas que pueden ofrecer a las Ciencias Cognitivas un modo de resistir el dominio del paradigma computacional, es decir, a la idea de que "la computadora parece ser el verdadero modelo de la inteligencia lógica, a la espera de un programa apropiado para acceder al atributo humano esencial de la racionalidad" (Dreyfus, 1972: 143), se cuenta la Fenomenología (junto con otras con la filosofía de Wittgenstein y Heidegger, así como la Psicología de la Gestalt). Si bien admite Dreyfus que estas concepciones introducen un grado de vaguedad experimental y menor rigurosidad científica a la investigación ${ }^{14}$, al menos, en comparación con las perspectivas tradicionales ancladas en las neurociencias y la biología, ofrecen conjeturas filosóficas más robustas. En opinión de Dreyfus, el aporte de la Fenomenología remediaría el descuido de tres dimensiones en la indagación de las Ciencias Cognitivas y la Inteligencia artificial sobre la cognición humana: el papel desempeñado por el cuerpo, la situación y las motivaciones (propósitos y necesidades) de los agentes. La conclusión de Dreyfus se ve reflejada en el párrafo que cito a continuación en el cual son comparados, en sus performances "cognitivas", un agente perceptivo humano y una máquina. "Una máquina - sostiene - puede, en el mejor de los casos, hacer un conjunto específico de hipótesis y luego averiguar si ellas han sido confirmadas o refutadas por los datos." El cuerpo, en cambio, cuenta con la capacidad de "modificar constantemente sus expectativas" las cuales se organizan y regulan en función de su interacción pasada y presente con el objeto. En consecuencia, "La relación con el objeto (coping) no necesita ser definida por un conjunto específico de atributos, sino más bien por un dominio (mastery) continuo, al cual Merleau-Ponty denomina máxima presa (maximum grasp)" (Dreyfus, 1972: 162). La concreción de la máxima presa depende del objetivo del agente y a su vez este se encuentra determinado por una situación en el contexto de la cual establece sus propósitos.

Por otra parte, como bien señala N. Depraz en el contexto de sus reflexiones sobre las consecuencias del proyecto de naturalización de la Fenomenología, el extremo 
reduccionismo que postula la explicación neurobiológica como última instancia de validación en la comprensión de la conciencia y la experiencia vivida, es algo que las Ciencias Cognitivas han tratado de superar en su desarrollo (Depraz, 1999: 474). Depraz identifica tres actitudes en este esfuerzo por tomar distancia del reduccionismo residual, (i) el cognitivismo computacional, (ii) el emergentismo y (iii) el emergentismo corporeizado o enactivo. A los fines de entender la evolución interna de las Ciencias Cognitivas estas actitudes se pueden entender como momentos en el desarrollo de la disciplina, aunque en rigor de verdad estos conviven, incluso en la actualidad, como programas de investigación alternativos. Como señala Depraz es posible reconocer en el paso del primer momento al segundo "una ruptura en el marco de referencia", mientras que el paso del segundo al tercero evidencia, más bien, una profundización del análisis de la propia posición emergentista. Este punto resulta de gran importancia en lo relativo al interés suscitado por Fenomenología de la Percepción en representantes de las Ciencias Cognitivas como Varela y Thompson y posteriormente por Gallagher, por ejemplo. El cognitivismo concibe la mente según el modelo de la computadora ${ }^{15}$, inspirado por el exitoso desarrollo de la cibernética, y avanza en la reducción de la cognición y los estados mentales, en general, a operaciones simbólicas gobernadas por reglas. Resulta interesante resaltar el fuerte componente dualista que subyace a esta concepción de lo mental como "dispositivo desencarnado de procesamiento de información entrante y saliente (input-output)" (Heiner, 2008: 118), intermediario privilegiado entre el mundo y las representaciones generadas a partir del procesamiento de la información. En ello reside, precisamente, el blanco principal de la crítica que dará origen al emergentismo. Esta sintética presentación del cognitivismo, lejos de pretender mostrarlo de manera simplificada, recoge los conceptos centrales que lo caracterizan. Como señala Depraz, el emergentismo "dudoso respecto de la ingenuidad (abstracción) de la traducción simbólica, presenta lo mental como una red de neuronas que interactúan localmente entre ellas, engendrando de ese modo un estado cognitivo global sub-simbólico cuyas propiedades intrínsecas propias no son reductibles a las propiedades locales de las neuronas" (Depraz, 1999: 474). El modelo de la computadora es sustituido así por la dinámica neuronal, siendo en consecuencia el cerebro el sustrato biológico de esas operaciones. El emergentismo, también llamado conexionismo, se enfoca en la relación de las neuronas que hacen posible la "emergencia" de los estados mentales y, en esta operación, se vuelven relevantes para el análisis las determinaciones contextuales y ambientales en las cuales se desarrolla la vida. Esto es lo que marca el cambio de marco de referencia intra-disciplinar que mencionaba Depraz, y que Hiener caracteriza como una mejora respecto del cognitivismo en cuanto menos tres aspectos: (i) la ampliación en la comprensión de la cognición como algo más que deducción lógica a partir de reglas y símbolos, es decir, la consideración de la cognición como inducción dependiente del contexto y del comportamiento (humano y no humano) situado, (ii) la introducción de las dimensiones temporal y contextual en la comprensión de la cognición no consideradas como variables de relevancia en la concepción computacional de lo mental y (iii) en relación con los dos aspectos anteriores, la posibilidad del "aprendizaje" de las redes neuronales bajo la condición de la retroalimentación entre el contexto y la experiencia de los sujetos, es decir, la superación de la comprensión cognitivista del aprendizaje como la aplicación discreta de reglas y representaciones predeterminadas y guardadas en la memoria (Heiner, 2008: 120). La perspectiva enactiva, por su parte, representa un avance en la superación del dualismo todavía subyacente en el emergentismo, al sustituir la centralidad de las representaciones por la acción. Para la perspectiva enactiva la dinámica del agente y el entorno (no solo biológico, sino

15 En otros términos, la presuposición de que la mente, como la computadora “....debe operar sobre datos completamente determinados de acuerdo a reglas estrictamente definidas" (Dreyfus, 1972: 154). 
también histórico y cultural) resulta fundamental en la explicación de la cognición, la cual es concebida como "resultado de una interpretación que emerge de nuestra capacidad de comprensión. Esta capacidad está arraigada en la estructura de nuestra corporización biológica, pero se vive y se experimenta dentro de un dominio de acción consensual e historia cultural. (Varela, Thompson y Rosch, 2005: 177).

\section{IV}

Sin intentar subordinar el esfuerzo filosófico de la fenomenología merleau-pontyana al naturalismo y reconociendo, además, que donde Merleau-Ponty encuentra una relación inestable de figura y fondo, perspectiva y campo fenomenal, el pensamiento objetivo reconoce relaciones causales y sensaciones o impresiones simples, una perspectiva de tercera persona o un pensamiento de sobrevuelo. Resulta, sin embargo, de fundamental importancia señalar que esta apreciación que vale, en general, para lo que Merleau-Ponty entiende como pensamiento objetivo, no caracteriza a todos los intentos de acercamiento de las Ciencias Cognitivas a la obra de Merleau-Ponty. Las Ciencias Cognitivas con las salvedades que se acaban de hacer y aún con sus pretensiones reduccionistas, se presentan en una continuidad descriptiva de segundo orden respecto de aquello a lo que la fenomenología tiene acceso inmediato, la experiencia. En este sentido, y en opinión de autores como Dreyfus y Gallagher, entre otros, es posible concebirlas como complementarias, antes bien que antagónicas. Lo que en este caso resulta imperioso prevenir es la transposición de los órdenes para no acabar reduciendo la experiencia a su correlato neurológico.

En algún sentido resulta lícito reconocer una analogía entre las investigaciones de las Ciencias Cognitivas y los resultados de la investigación empírica proveniente de la Psicología que inundan las páginas de Fenomenología de la Percepción. Mutatis mutandis las Ciencias Cognitivas contemporáneas parecen ser la Psicología de la época en que Merleau-Ponty escribía sus obras. ${ }^{16}$ En consecuencia, seguir en la línea de investigación anunciada por Merleau-Ponty no debería, al menos en principio, implicar un tajante rechazo de los hallazgos y desarrollos de las Ciencias Cognitivas, más bien por el contrario, representaría un incentivo para acercarnos a ese campo de investigación con las herramientas adecuadas y los recaudos metodológicos ya indicados por el propio filósofo en Fenomenologia de la Percepción. 


\section{Bibliografía}

"Brook, A. (2008). Phenomenology: Contribution to Cognitive Science. Abstracta. Linguagem, Mente e Ação, Special Issue II, 54-70.

"Depraz, N. (1999). When Transcendental Genesis Encounters the Naturalization Project. En Roy, J. M., Petitot, J., Pachoud, B. y Varela, F. (Eds.), Naturalizing Phenomenology: Issues in Contemporary Phenomenology and Cognitive Science (pp. 464489). Stanford: Stanford University Press.

» Gallagher, S. (2008) Cognitive Science. En Diprose, R. y Reynolds, J. (Eds.), Merleau-Ponty: Key Concepts (pp. 207-217), London-New York: Routledge, Taylor and Francis.

"Gardner, S. (2015) Merleau-Ponty's Transcendental Theory of Perception. En Gardner, S. and Grist, M. (Eds.), The Transcendental Turn (pp. 294-323), Oxford: Oxford University Press.

»Heiner, B. T. (2008). The recorporealization of cognition in phenomenology and cognitive science. Continental Philosophy Review, 41, 115-126.

"Husserl, E. (1991) La Crisis de las Ciencias Europeas y la Fenomenología Trascendental, Trad. J. Muñoz y S. Mas. Barcelona: Crítica.

" Madison, G. B. (1990). Merleau-Ponty et la déconstruction du logocentrisme. Laval théologique et philosophique, 46, 1, 65-79.

» Merleau-Ponty, M. (1964). Signos (trad. C. Martínez y G. Oliver). Barcelona: Seix Barral.

» Merleau-Ponty, M. (1985). Fenomenología de la Percepción (trad. J. Cabanes). Barcelona : Espasa Calpe. (Phénoménologie de la Perception. Paris: Gallimard, 1945).

» Merleau-Ponty, M. (2000) Titres et Travaux. Projet d`enseignement. En Parcours deux (pp. 9-35). Paris: Verdier.

»Petitot, J., Varela, F., Pachoud, B., Roy, J.-M. (1999) Naturalizing Phenomenology. Issues in Contemporary and Cognitive Science, Stanford, California: Stanford University Press.

"Pollard, C. (2014). Merleau-Ponty and Embodied Cognitive Science. Discipline Filosofiche, XXIX 2, 67-90.

"Varela, F. (2000). El fenómeno de la vida. Santiago: Dolmen Ediciones.

"Varela, F., Thompson, E. y Rosch, E. (2005). De cuerpo presente (trad. C. Gardini). Barcelona: Gedisa.

"Walton, R. (2012). El sentido de la construcción en la fenomenología de MerleauPonty. En Ramírez, M. (coord.) Merleau-Ponty viviente (pp. 137-152). Barcelona: Anthropos. 